\title{
SAS and SSA Conditions for Congruent Triangles
}

\author{
Alexander F. Mironychev \\ Challenge Early College HS, 5801 West Loop South Freeway, Houston, TX 77081, USA
}

\begin{abstract}
For two triangles to be congruent, SAS theorem requires two sides and the included angle of the first triangle to be congruent to the corresponding two sides and included angle of the second triangle. If the congruent angles are not between the corresponding congruent sides, then such triangles could be different. It turns out that it is possible to describe four cases in which triangles are congruent even though congruent angles. For two triangles to be congruent, SAS theorem requires two sides and the included angle of the first triangle to be congruent to the corresponding two sides and included angle of the second triangle. If the congruent angles are not between the corresponding congruent sides, then such triangles could be different. It turns out that it is possible to describe four cases in which triangles are congruent even though congruent angles are not between the corresponding congruent sides. Such a theorem could be named, for example, SSA theorem. Many texts state that two triangles cannot be shown to be congruent if the condition of SSA exists. However, the author describes cases in which such triangles could be proven congruent with the SSA theorem. An immediate consequence of this new understanding is the necessity of revising many problems and answers in high school and college-level texts related to congruent triangles.are not between the corresponding congruent sides. Such a theorem could be named, for example, SSA theorem. An immediate consequence of this new understanding is the necessity of revising many problems and answers in high school and college-level texts related to congruent triangles.
\end{abstract}

Key words: Congruent triangles, a theorem, a proof, superposition, SAS and SSA conditions.

\section{Introduction}

The current paper decsusses the congruency of triangles under SSA conditions. In the traditional high school Geometry courses [1], [2] there are five basic statements about congruent triangles. They are SAS, SSS, ASA, AAS, and HL statemnts. As usual, statements SAS, ASA, and SSS are considered as postulates. Statements AAS and HL are considered as theorems. In curricum of other countries, for example, in Russian high school curriculum all these statements are considered as theorems [3]. The special case is SSA conditions when congruent angle is not between congruent sides. Under such conditions it could occur triangles will be evidently uncongruent. In the case of right triangles, SSA conditions do lead to the conguency - this is the well known HL theorem. The question under consideration is the existence of

Corresponding author: Alexander F. Mironychev, Ph.D., research field: educational mathematics and the mathematical economics. congruency when triangles satisfy SSA conditions and are not right triangles.

Results of this research were reported at the at Sam Houston State University (Huntsville, 2015) [4], at Houston ISD (Houston, 2017), and at the MAA Joint Meeting (San Diego, 2018).

\section{Proofs and Definitions}

In the educational practice there are different types of proofs. The most commonly used proofs are: (1) paragraph proof; (2) two column proof; and (3) chart proof. (Wikipedia, and something else). The proofs itself could be made either on the basis of some previous theorems or lemmas, or on the basis of certain definitions, or on the basis of combination of previous theorems and definitions. For the concept of conguency often is used the following

Definition 1. Triangles (figures) are congruent if they have the same sides and the same angles.

Such definition is widely used in high school Geometry courses. It can be used and is being used for 
proofs of theorems about congruent triangles. For example, it is used for the proof of SAA and HL theorems [1, 2]. As for statements SAS, ASA, and SSS, they are considered in these books as postulates despite that in Euclid [5] and many other Geometry courses $[3,6]$ they are considered as theorems. These statements hardly can be proved as theorems on the basis of the Definition 1. To make it possible it is necessary to accept more effective definition. The main idea here is the superposition of figures. Superposition means the motion of figures and matching them on each other. To deside whether figures are congruent they have be completely matched. If they coniside in all points, then they are considered as congruent (equal). Such concept of congruency is used in Geometry for a long time. For this reason in the current paper let us acceprt such more effective.

Definition 2. Triangles (figures) are congruent if they coinside in all points after they match on each other.

In such case the Definition 1 also can be used but as a corollary or as an explanation of the Definition 2. In other words, if trangles coinside in all points, then in accordance with the Definition 1 they are congruent that means that in such triangles corresponding sides and corresponding angles are the same.

It is necessary to mention that triangles (figures) can be matched by one of three main basic transformations or by the combination of them. These basic transformations are:

(1) translation in some direction-right, left, up, or down picture

(2) rotation-clockwise or counterclockwise around some point of rotation picture

(3) reflection across some axis of reflection picture

(4) combination of all previos three transformations.

So, the proof of congruentcy implies to move figures on each other and then describe that these figures coinside in all points. Such proofs are well known since Euclid and is successfully used in many textbooks on Geometry [6].

\section{SAS and SSA Conditions for Congruent Triangles}

SAS statement says that two triangles are congruent if two sides and the included angle in one triangle are congruent to two sides and the included angle in another triangle. Such case is represented in Fig.1. This statement as a theorem was proved in Greek time [5]. This proof is still used in Geometry courses [3, 6]. The proof is based on the super-position of triangles and on the postulate that there is only one line passing through two given points.

Important point is that the congruent angle lies between congruent sides. Under SAS conditions triangles are always congruent. Now let us consider the case when congruent angels are not between congruent sides. Name such conditions as SSA conditions. The question under consideration is-can triangles be congruent in such case? There is some literature discussing this question. Many sites in Internet demonstrate pictures related to this statement and possible consequences whether triangles look congruent or not congruent. Unfortunately such sites are subject to changes and often disapper from the web. It is easy to see that such conditions could describe the case when two triangles are evidently not congruent-see Fig. 3.

Some theoretical analysis such cases was done by $\mathrm{N}$. Rozov from Moscow State University [7]. He also represented the existence of the congruency with SSA conditions in one special case. Another example of the existence of the conguent triangles with SSA conditions was done by A. Bogomolny [8]. On the web site created by Donna Roberts [9] there are examples and assignments related to cases with congruent and not congruent triangles which still satisfy SSA conditions. These conditions are called "Donkey Theorem".

Some authors suggest that the existence of congruency can be true only for right triangles [2]. It is very attractive to describe all possible cases with triangles that satisfy SSA conditions and to get a final 
answer for the question about the congruency of such triangles.

\section{SSA Theorem}

Let us prove the following

Theorem 1. Let in two triangles there are two congruent sides and one angle. The congruent angles are not betwen congruent sides. If other corresponding angles are both acute or obtuse, then triangles are congruent.

There are six possible combinations of sides and angles for this theorem:

(1) Congruent angles $\angle A$ and $\angle A^{\prime}$ in both triangles are acute; the included angle $\angle C$ in the first triangle $\triangle A B C$ is obtuse and the included angle $\angle C$ ' in the second triangle $\angle A^{\prime} B^{\prime} C^{\prime}$ is acute; the third angle $\angle B$ in the first triangle $\triangle A B C$ is acute and the third angle $\angle B^{\prime}$ in the second triangle $\triangle A^{\prime} B^{\prime} C^{\prime}$ is obtuse. This case is represented in Fig. 1.

(2) Congruent angles $\angle A$ and $\angle A^{\prime}$ in both triangles are acute; the included angle $\angle C$ in the first triangle $\triangle A B C$ is acute and the included angle $\angle C$ ' in the second triangle $\angle A^{\prime} B^{\prime} C^{\prime}$ is obtuse; the third angle $\angle B$ in the first triangle $\triangle A B C$ is obtuse and the third angle $\angle B^{\prime}$ in the second triangle $\triangle A^{\prime} B^{\prime} C^{\prime}$ is acute. Such triangles are shown in Fig. 2.

(3) Fig. 3 describes triangles when congruent angles $\angle A$ and $\angle A^{\prime}$ in both triangles are acute; the included angles $\angle C$ and $\angle C$ ' in both triangles are obtuse; and the third angles $\angle \mathrm{B}$ and $\angle B$ ' in both triangles are acute;

(4) In Fig. 4, congruent angles $\angle A$ and $\angle A^{\prime}$ in both triangles are acute; the included angles $\angle C$ and $\angle C$ ' in both triangles are acute; and the third angles $\angle B$ and $\angle B^{\prime}$ in both triangles are acute;

(5) Fig. 5 shows the case when congruent angles $\angle A$ and $\angle A^{\prime}$ in both triangles are acute; the included angles $\angle C$ and $\angle C$ ' in both triangles are acute; and the third angles $\angle B$ and $\angle B$ ' in both triangles are obtuse;
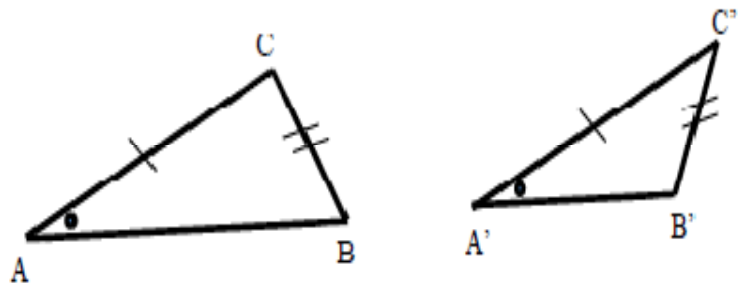

Fig. 1 Triangles that satisfy SSA conditions.
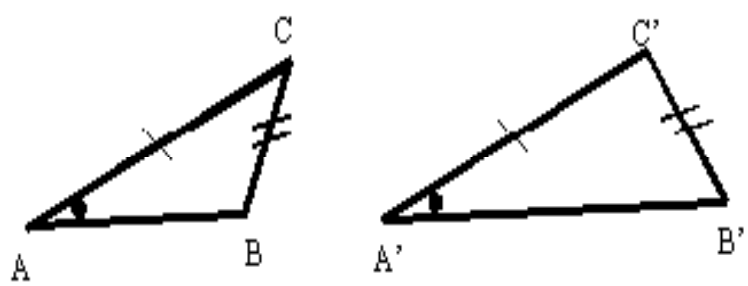

Fig. 2 Triangles that satisfy SSA conditions.
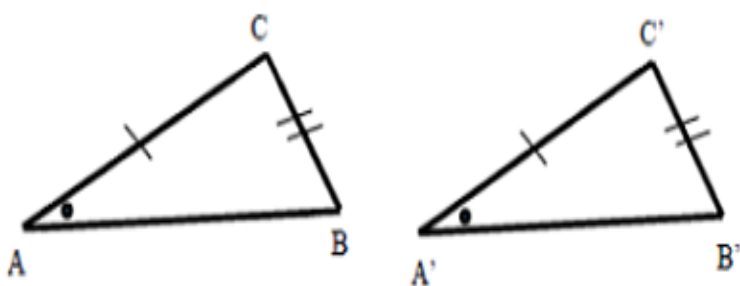

Fig. 3 Triangles that satisfy SSA conditions.
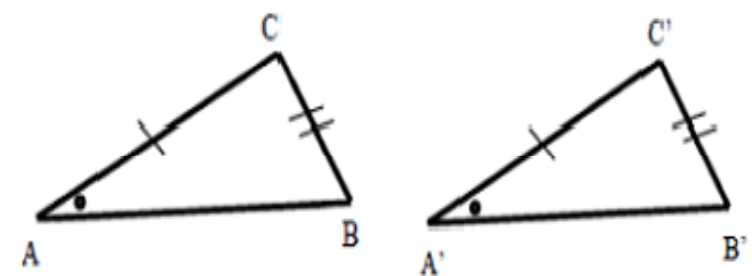

Fig. 4 Triangles that satisfy SSA conditions.
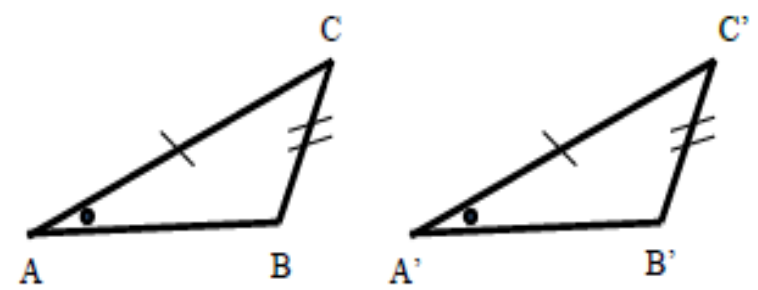

Fig. 5 Triangles that satisfy SSA conditions.

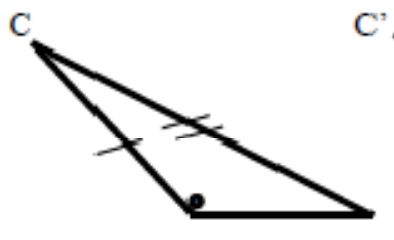

A

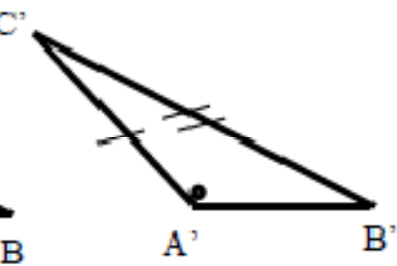

A
Fig. 6 Triangles that satisfy SSA conditions. 
Table 1 Possible cases with SSA conditions.

\begin{tabular}{lllll}
\hline Type & Congruent angles & Included angles & Third angles & Congruent triangles \\
\hline 1 & Acute & Obtuse & Acute & No \\
2 & Acute & Acute & Obtuse & No \\
3 & Acute & Obtuse & Acute & Yes \\
4 & Acute & Acute & Obtuse & Yes \\
5 & Acute & Acute & Acute & Yes \\
6 & Obtuse & Acute & Acute & Yes \\
\hline
\end{tabular}

(6) In Fig. 6 congruent angles $\angle A$ and $\angle A$ ' in both triangles are obtuse; the included angles $\angle C$ and $\angle C^{\prime}$ ' in both triangles are acute; and the third angles $\angle B$ and $\angle B^{\prime}$ in both triangles are acute.

All these cases are represented in Table 1.

It is evident that in cases 1 and 2 triangles cannot be congruent because they have different angles. As usual only these cases are discussed in literature to show that SSA theorem is inconsistent. But it turns out that in four next cases 4-6 triangles are congruent. To prove the congruency in all these four above cases, it necessary to use

- the concept of the superposition of figures;

- the Definition 2 of congruency, and

- well known property of isosceles triangles:

Statement 1. In the isosceles triangle, the base angles are acute and congruent.

In this paper we omit the proof of this statement because it is available almost in any Geometry textbook.

Proof of the Theorem 1:

- Consider the case 3 from Table 1.

Given are two congruent triangles $\triangle A B C$ and $\triangle A^{\prime} B^{\prime} C^{\prime}$ with two congruent sides, the congruent acute angles, and with the included obtuse angles, and with the third acute angles.

Let us match $\triangle A B C$ onto $\triangle A^{\prime} B^{\prime} C^{\prime}$ as is represented in Fig. 7.

Side $A C$ will coincide in all points with the side $A^{\prime} C^{\prime}$ ' because they are congruent. Side $A B$ will go along $A^{\prime} B^{\prime}$ 'because the angle $\angle C A B$ is congruent to the angle $\angle C^{\prime} A^{\prime} B^{\prime}$. Pretend that sides $C B$ and $C^{\prime} B^{\prime}$ ' do not coincide and, for example, the side $C^{\prime} B^{\prime}$ ' is inside the triangle $\Delta\left(A A^{\prime}\right)\left(C C^{\prime}\right) B$. Then the triangle $\triangle B^{\prime}\left(C C^{\prime}\right) B$ will be an isosceles triangle. Once angle $\angle\left(C C^{\prime}\right) B^{\prime}\left(A A^{\prime}\right)$ is acute, then the angle $\angle\left(C C^{\prime}{ }^{\prime}\right) B^{\prime} B$ must be obtuse. In this case in the isosceles triangle $\triangle B^{\prime}\left(C C^{\prime}\right) B$ one base angle is acute and the second base angle is obtuse. Such outcome is impossible. So, the suggestion that sides $C B$ and $C$ ' $B$ ' do not coincide is inconsistent. On the opposite, these sides must coincide. It means that triangles $\triangle A B C$ and $\triangle A^{\prime} B^{\prime} C^{\prime}$, coincide in all points. In other words, these triangles are congruent.

- Consider the case 4 from Table 1.

Given are two congruent triangles $\triangle A B C$ and $\triangle A^{\prime} B^{\prime} C^{\prime}$, with two congruent sides, the congruent acute angles, and with the included acute angles, and with the third obtuse angles.

Let us match $\triangle A B C$ onto $\triangle A^{\prime} B^{\prime} C^{\prime}$ as is represented in Fig. 8.

Side $A C$ will coincide in all points with the side $A^{\prime} C^{\prime}$ ' because they are congruent. Side $A B$ will go along $A^{\prime} B^{\prime}$ because the angle $\angle C A B$ is congruent to the angle $\angle C^{\prime} A^{\prime} B^{\prime}$. Pretend that sides $C B$ and $C^{\prime} B^{\prime}$ ' do not coincide and, for example, the side $C^{\prime} B^{\prime}$ ' is inside the triangle $\triangle\left(A A^{\prime}\right)\left(C C^{\prime}\right) B$. Then the triangle $\triangle B^{\prime}\left(C C^{\prime}\right) B$ will be an isosceles triangle. Once angle $\angle\left(C C^{\prime}\right) B^{\prime}\left(A A^{\prime}\right)$ is obtuse, then the angle $\angle\left(C C^{\prime}\right.$ ') $B$ ' $B$ must be acute. In this case in the isosceles triangle

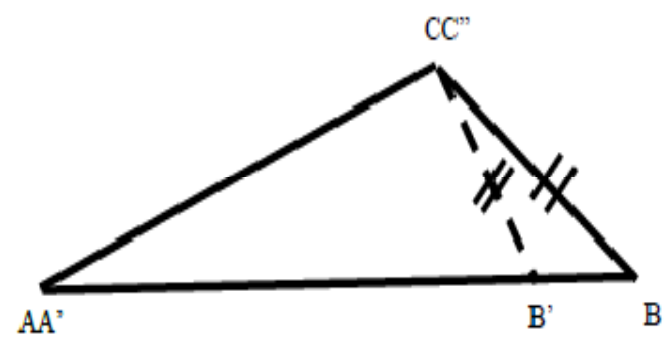

Fig. 7 Proof of the case 3. 


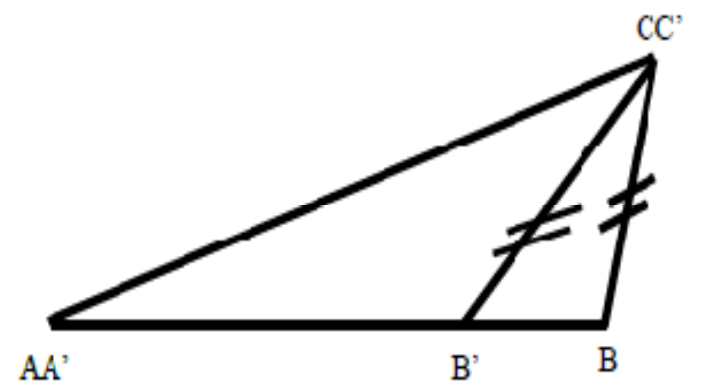

Fig. 8 Proof of the case 4.

$\triangle B^{\prime}\left(C C^{\prime}\right) B$ one base angle is acute and the second base angle is obtuse. Such outcome is impossible. So, the suggestion that sides $C B$ and $C$ ' $B$ ' do not coincide is inconsistent. On the opposite, these sides must coincide. It means that triangles $\triangle A B C$ and $\triangle A^{\prime} B^{\prime} C^{\prime}$ ' coincide in all points. In other words, these triangles are congruent.

- Consider the case 5 from Table 1.

Given are two congruent triangles $\triangle A B C$ and $\triangle A^{\prime} B^{\prime} C^{\prime}$ ' with two congruent sides, the congruent acute angles, and with the included acute angles, and with the third obtuse angles.

Let us match $\triangle A B C$ onto $\triangle A^{\prime} B^{\prime} C^{\prime}$ as is represented in Fig. 9.

Side $A C$ will coincide in all points with the side $A^{\prime} C^{\prime}$ because they are congruent. Side $A B$ will go along $A^{\prime} B^{\prime}$ 'because the angle $\angle C A B$ is congruent to the angle $\angle C^{\prime} A^{\prime} B^{\prime}$. Pretend that sides $C B$ and $C^{\prime} B^{\prime}$ do not coincide and, for example, the side $C^{\prime} B^{\prime}$ ' is inside the triangle $\triangle\left(A A^{\prime}\right)\left(C C^{\prime}\right) B$. Then the triangle $\triangle B^{\prime}\left(C C^{\prime}\right) B$ will be an isosceles triangle. Once angle $\angle\left(C C^{\prime}\right) B^{\prime}\left(A A^{\prime}\right)$ is obtuse, then the angle $\angle\left(C C^{\prime \prime}\right) B^{\prime} B$ must be acute. In this case in the isosceles triangle $\triangle B^{\prime}\left(C C^{\prime}\right) B$ one base angle is acute and the second base angle is obtuse. Such outcome is impossible. So, the suggestion that sides $C B$ and $C$ ' $B$ ' do not coincide is inconsistent. On the opposite, these sides must coincide. It means that triangles $\triangle A B C$ and $\triangle A^{\prime} B^{\prime} C^{\prime}$ ' coincide in all points. In other words, these triangles are congruent.

- Consider the case 6 from Table 1.

Given are two congruent triangles $\triangle A B C$ and $\triangle A^{\prime} B^{\prime} C^{\prime}$ with two congruent sides, the congruent acute angles, and with the included acute angles, and with the third obtuse angles.

Let us match $\triangle A B C$ onto $\triangle A^{\prime} B^{\prime} C^{\prime}$ as is represented in Fig. 10.

Side $A C$ will coincide in all points with the side $A^{\prime} C^{\prime}$ because they are congruent. Side $A B$ will go along $A^{\prime} B$ ' because the angle $\angle C A B$ is congruent to the angle $\angle C^{\prime} A^{\prime} B^{\prime}$. Pretend that sides $C B$ and $C^{\prime} B^{\prime}$ ' do not coincide and, for example, the side $C^{\prime} B^{\prime}$ ' is inside the triangle $\triangle\left(A A^{\prime}\right)\left(C C^{\prime}\right) B$. Then the triangle $\triangle B^{\prime}\left(C C^{\prime}\right) B$ will be an isosceles triangle. Once angle $\angle\left(C C^{\prime}\right) B^{\prime}\left(A A^{\prime}\right)$ is acute, then the angle $\angle\left(C C^{\prime}{ }^{\prime}\right) B^{\prime} B$ must be obtuse. In this case in the isosceles triangle $\triangle B^{\prime}\left(C C^{\prime}\right) B$ one base angle is acute and the second base angle is obtuse. Such outcome is impossible. So, the suggestion that sides $C B$ and $C$ ' $B$ ' do not coincide is inconsistent. On the opposite, these sides must coincide. It means that triangles $\triangle A B C$ and $\triangle A^{\prime} B^{\prime} C^{\prime}$ coincide in all points. In other words, these triangles are congruent. So, in all cases 4-6 triangles are congruent.

End of the Theorem 1.

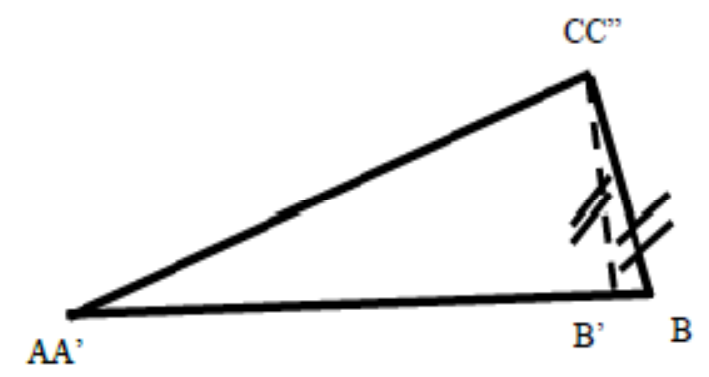

Fig. 9 Proof of the case 5.

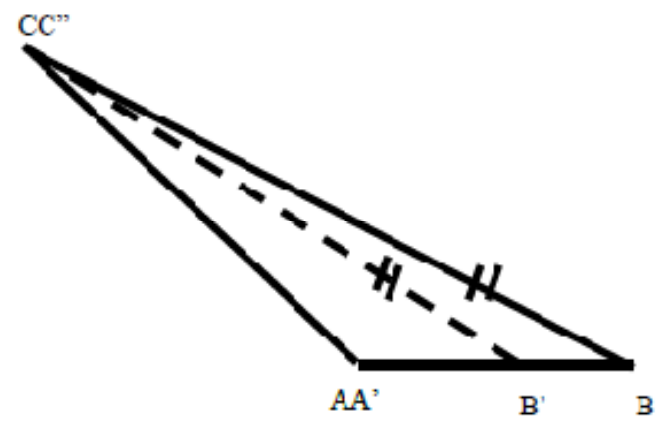

Fig. 10 Proof of the case 6. 


\section{Discussion of the Theorem}

It seems that the SSA theorem could be a supplement for the traditional set of theorems about congruent triangles along with SAS, ASA, AAS, SSS, and HL statements. In each triangle there are six parts-three sides and three angles. Actually the congruence means the comparison of all these six parts in triangles under consideration. But the known five congruence theorems require to get into considerations only three parts - two sides and angle (SAS), or two angles and one side (ASA and AAS), or three sides (SSS). The HL theorem requires two congruent elements which are the hypotenuse and on angle. But the proof of this theorem implies the existence of one right angle in each compared triangle. So, the HL theorem also requires the congruency of three elements in each triangle.

The SSA theorem also requires three congruent elements in each triangle which are two sides and one angle - the same amount of information as in the SAS theorem. But the SSA theorem also needs the additional quantitative knowledge about the next two angles. In any case this information is still less than the knowledge about all six elements in each triangle. So, the SSA theorem could be considered as a scientific significance. In literature there are some more statements that describe conditions for congruent triangles. They refer to the:

- comparison of one side, one angle, the angle bisector [3, 10];

- comparison of two sides and the median [10];

- comparison of two sides and the median [10];

- sides with bisectors, medians, and altitudes in triangles under consideration.

But such statements mainly are used for educational purposes es exercises for proofs.

\section{Application of the Theorem}

The theorem proved above urges to review and to reevaluate answers for many old problems discussed in textbooks. For example, consider some problems from Geometry textbook by McDougal Littell [1] which is widely used in the high school Geometry courses.

- Page 243, Example 9.

QUESTION: Decide whether information is given to prove that the triangles are congruent using the SAS congruence postulate.

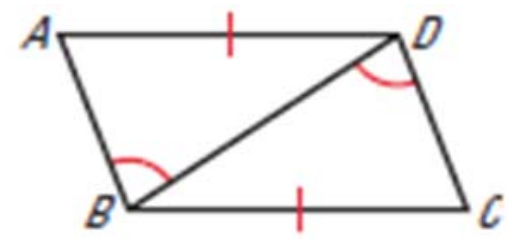

The correct answer for the given question is NO. The SAS statement (postulate or theorem) cannot be applied because the given congruent angles $\angle B D C$ and $\angle A B D$ are not between congruent sides $B C$ and $A D$ and the common side $B D$. The question whether given triangles $\triangle B D A$ and $\triangle D B C$ are congruent remains uncertain. But from the picture it follows that other angles in the given triangles are acute. It is exactly the case 5 from SSA theorem. So, triangles $\triangle B D A$ and $\triangle D B C$ are congruent.

- Page 243, Example 11.

QUESTION: Decide whether information is given to prove that the triangles are congruent using the SAS congruence postulate.

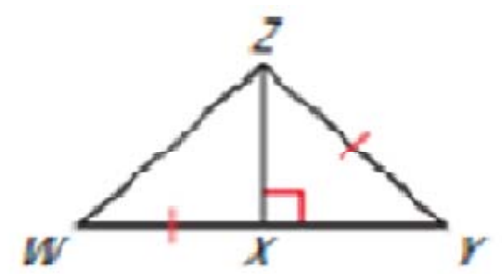

The correct answer for the given question is NO. The SAS statement (postulate or theorem) cannot be applied because the right congruent angles $\angle W X Z$ and $\angle Y X Z$ are not between congruent sides $Z Y, W X$, and the common side $Z X$. More precisely, the right angle $\angle W X Z$ is the included angle between the side $W X$ which is congruent to the side $Z Y$ and the common side $Z X$ in the triangle $\triangle W X Z$. In the $\triangle Y X Z$, the right angle $\angle Y X Z$ is not the included angle between the 
congruent side $Y Z$ and the common side $X Z$. The SSA theorem cannon be applied neither because of the same reason. Moreover, it is evident the figure is not drawn in the scale. In congruent triangles the hypotenuse (in our case it is the side $Y Z$ ) cannot be congruent the leg (in our case it is the side $W X$ ). So, such triangles are certainly incongruent.

- Page 243, Example 12.

QUESTION: Decide whether information is given to prove that the triangles are congruent using the SAS congruence postulate.

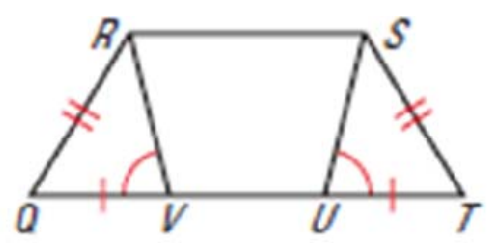

The correct answer for the given question is NO. The SAS statement (postulate or theorem) cannot be applied because the given congruent angles $\angle R V Q$ and $\angle S U T$ are not between congruent sides $R Q$ and $V Q$ in the triangle $\triangle Q V R$ and congruent sides $S T$ and $U T$ in the triangle $\triangle T U S$. The question whether given triangles $\triangle Q V R$ and $\triangle T U S$ are congruent remains uncertain. But from the picture it follows that other angles in the given triangles are acute. It is exactly the case 5 from SSA theorem. So, triangles $\triangle Q V R$ and $\triangle T U S$ are congruent.

- Page 243, Example 14.

QUESTION: Decide whether information is given to prove that the triangles are congruent using the SAS congruence postulate.

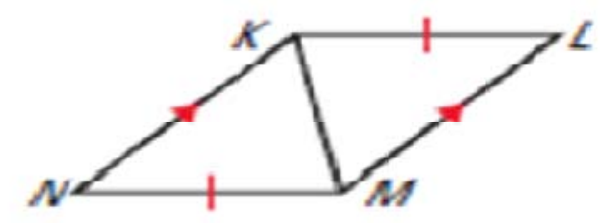

The correct answer for the given question is NO. The SAS statement (postulate or theorem) cannot be applied for triangles $\triangle N M K$ and $\triangle L K M$. These triangles have congruent sides $M N$ and $K L$ and the common side $K M$. In these triangles also there are congruent angles $\angle N K M$ and $\angle L M K$ as alternate interior angles between parallel lines $N K$ and $M L$. But these angles are not included angles between congruent sides. So, the SAS congruency statement is not applicable. The question whether given triangles $\triangle N M K$ and $\triangle L M K$ are congruent remains uncertain. But from the picture it follows that other angles in the given triangles are acute. It is exactly the case 5 from SSA theorem. That gives the opportunity to conclude that triangles $\triangle N M K$ and $\triangle L M K$ are congruent.

- Page 244, Example 20.

QUESTION: Decide whether enough information is given to prove that the triangles are congruent. If there is enough information, state the congruence postulate or theorem you should use.

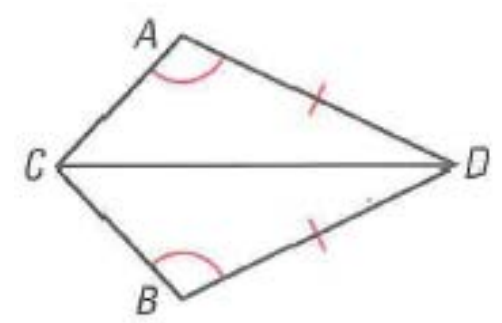

With the set of traditional statements the correct answer is going to be NO. But it is the case 6 of the SSA theorem proved above. So, now the answer for this problem should be YES. The given triangles are congruent.

\section{Conclusions}

The SSA theorem can be included into the set of theorems about congruent triangles. This theorem enables to recognize the congruency in many problems where the traditional set of theorems leaves the final answer uncertain. In the light of these results it makes sense to review answers for many old problems related to congruent triangles.

\section{Acknowledgements}

Author thanks Sam Houston University and Houston ISD for the support of his efforts. This investigation was discussed with my colleagues Mr. Warren Morales, Mr. Charles Burrus, and Ms. Gloria 
Carrillo who also spoke out valuable opinions. Unvaluable help was received from Dr. Igor Kliakhlander.

\section{References}

[1] Larsen, R., Boswell, L., and Stiff, L. 2007. Geometry. McDougal Littell.

[2] Edward, B. B., et al. 2007. Geometry. Holt, Rinehart and Winston.

[3] Atanasian, L. S., Butuzov, V. F., Kadomzev, C. B., and Pozniak, E. G. 2015. Geometria. Moscow: Prosveshenie. (in Russian).

[4] Mironychev, A. F. 2015. "Congruency Theorems in the Geometry Curriculum of High Schools: An International Comparison." In Proceedings of the International Conference "Universality of Global Education Issues", vol.2, Sam Houston State University, Retrieved from http://digital.library.shsu.edu/cdm/compoundobject/collec tion/p16042coll4/id/56.

[5] Euclid. 2016. The Elements. vol. 1, Dover.

[6] Eugine, S. F. 2002. Plane Geometry. A Beka Book.

[7] Rozov, N. 2010. "Chetverty Priznak Ravenstva Treugolnikov.” $\quad$ Retrieved from http://school.msu.ru/2010-09-22-13-34-23/37-2010-04-19 -12-46-37/59-2010-04-19-12-50-14. (in Russian)

[8] Bogomolny, A. 2017. "The SSA." Retrieved from https://www.cut-the-knot.org/pythagoras/SSA.shtml.

[9] Roberts, D. 2012. "Regents Exam Preparation Center." Retrieved from http://regentsprep.org/regents/math/geometry/gp4/ltriangl es.htm.

[10] Pogorelov, A. V. 2001. Geometria. Moscow: Prosveshenie. (in Russian) 